\title{
REPRESENTAÇÃO GRÁFICA ARQUITETÔNICA DIGITAL: AVALIAÇÃO DO USO DE NOVAS ABORDAGENS DIDÁTICAS PARA MELHORIA NO PROCESSO DE ENSINO-APRENDIZAGEM
}

\author{
Vinícius Albuquerque Fulgêncio \\ Universidade Federal de Pernambuco \\ vinicius.fulgencio@ufpe.br \\ Andiara Valentina de Freitas e Lopes \\ Universidade Federal de Pernambuco \\ andiara.lopes@ufpe.br \\ Mariana Buarque Ribeiro de Gusmão \\ Universidade Federal de Pernambuco \\ mariana.rgusmao@ufpe.br
}

\section{DIGITAL ARCHITECTURAL GRAPHIC REPRESENTATION: EVALUATION OF THE USE OF NEW DIDACTIC APPROACHES FOR TEACHING-LEARNING PROCESS IMPROVEMENT}

\section{Resumo}

A representação da forma é imprescindível para qualquer área que envolva criação de artefatos, uma vez que sem ela o projeto não passa de uma ideia no campo mental. Porém para que a ideia seja materializada é preciso representá-la graficamente. Para isso é necessário que o projetista tenha capacidade de visualização espacial desenvolvida, o que tem se mostrado ser uma tarefa complexa. Ao longo da história, educadores têm se debruçado sobre estratégias didático-metodológicas a fim de ajudar nesse processo. Entre eles, Tamashiro defende que o ensino de desenho arquitetônico deve trabalhar com o uso do Conhecimento Técnico-Construtivo (CTC) para um melhor desenvolvimento das habilidades de representação. O presente trabalho toma como problema de pesquisa as dificuldades de visualização espacial dos estudantes no processo de representação gráfica do projeto arquitetônico. Foi realizado um experimento didático utilizando o CTC com estudantes de Arquitetura e Urbanismo, da Universidade Federal de Pernambuco. $O$ experimento exigiu que os discentes realizassem o levantamento de escadas e representassem graficamente suas projeções mongeanas em meio digital, utilizando-se das regras do desenho técnico. Os resultados mostram que o CTC contribuiu para o desenvolvimento da capacidade de visualização espacial e, consequentemente, para o processo de projeto e suas representações gráficas.

Palavras-chaves: ensino de arquitetura, representação gráfica para arquitetura, capacidade técnico construtiva, capacidade visiográfica tridimensional.

\begin{abstract}
A project without its graphic representation is an idea in the mental field. In order to accomplish this representation the development of spatial visualization is necessary, and it is a complex task. Throughout history, educators have been focusing on didacticmethodological strategies in order to assist in this process. Among these scholars,
\end{abstract}


Tamashiro argues that with the intention of having a better development of representation abilities the technical-constructive knowledge (TCK) method should be used to teach architectural drawing. The subject of this paper approaches the difficulties experienced by students during the process of making architectural drawings. A didactic experiment was carried out using the TCK with students of Architecture and Urbanism at Federal University of Pernambuco. The experiment required students to carry out a measurement of an existent stair and graphically represent its orthogonal projections using computational tools and technical drawing rules. The results show that TCK contributed to the development of spatial visualization capacity and, consequently, to the architectural drawing process.

\section{Keywords: teaching of architecture, architectural graphical representation, technical constructive knowledge, visiographic language}

\section{Introdução}

A representação da forma é imprescindível para qualquer área que envolva criação de artefatos, uma vez que sem ela o projeto não passa de uma ideia no campo mental. Porém para que uma ideia seja materializada é preciso que ela passe pela fase de projeto. Atualmente os projetos de quaisquer artefatos envolvem a passagem de ideias de objetos tridimensionais para representações bidimensionais e vice-versa. Essa dinâmica envolve a capacidade de visualização espacial, que é uma habilidade cognitiva que precisa ser desenvolvida e treinada. Geralmente os estudantes das áreas de Arquitetura, Engenharia, Design passam por um treinamento para desenvolver essa habilidade. Todavia, desenvolver a capacidade de visualização espacial não é uma tarefa fácil. Assim os profissionais dessas áreas precisam conhecer as regras dos sistemas de representação para transformar a ideia em algo que possa ser executado (Montenegro, 2007).

Ao tratarmos da representação gráfica aplicada à Arquitetura é comum que leigos associem tais representações à desenhos ilustrativos os quais são utilizados para comunicar a ideia do projeto ao cliente. Já no meio técnico é habitual se fazer uma relação com os desenhos técnicos (planta baixa, cortes e fachadas) comumente associados à área para a execução do projeto (Ching, 2012). Nesse sentido, é possível afirmar que a linguagem do arquiteto é o desenho e suas relações geométricas. Dessa maneira, a representação gráfica se refere ao conjunto de métodos utilizados para expressar ideias e conceitos, desde às mídias mais tradicionais (desenho a lápis), aqui tratadas como mídias analógicas, até as mídias digitais computacionais e de realidade virtual (Farelly, 2014). No entanto, os processos de representação dos projetos arquitetônicos se colocam limitados por não conseguirem representar de maneira completa o espaço arquitetônico (Zevi, 1996). Pode-se dizer ainda que a maior lacuna do desenho arquitetônico está ligada à sua dependência com relação a capacidade da visualização em síntese das partes do projeto (Montenegro, 2001).

A capacidade de visualizar objetos, compreendê-los e manipulá-los graficamente é definida por Rêgo (2008) como Capacidade Visiográfica Tridimensional (CV3D), sendo a representação gráfica sua principal ferramenta mediadora. O conceito definido por Rêgo (2008) é:

[...] habilidade cognitiva de perceber e compreender formas tridimensionais e expressá-las graficamente em representações bidimensionais (perspectivas e vistas ortográficas) e tridimensionais (maquetes e modelos geométricos), 
que podem incorporar ou não uma linguagem gráfica sistematizada (Rêgo, 2008, p. 44).

A CV3D, segundo Rêgo (2008), caracteriza-se pelo desenvolvimento de três habilidades: 1) observar uma forma tridimensional e representá-la graficamente; 2) imaginar e visualizar formas mentalmente e representá-las graficamente por meio analógico ou digital e; 3) observar uma representação gráfica tridimensional e representá-la em 2D e 3D a partir de outro ponto de vista. Portanto, entende-se que o problema real consiste em como desenvolver/ensinar a capacidade visiográfica.

Há muitos anos diversos profissionais se debruçam sobre esse problema e, mais especificamente, Tamashiro (2010) que defende que o ensino de desenho arquitetônico deve, dentre outros aspectos, trabalhar com o Conhecimento Técnico-Construtivo (CTC) para um melhor desenvolvimento das habilidades de representação, ou seja, para um melhor desenvolvimento da CV3D. Segundo o estudo desenvolvido por Tamashiro (2010), os discentes apresentam dificuldades quanto à capacidade de representação do projeto arquitetônico por diversas razões, dentre elas está o pouco conhecimento das projeções e regras da geometria descritiva, devido à redução da carga horária das disciplinas que tratam desses conteúdos; bem como pelo desconhecimento de uma série de elementos construtivos no mundo concreto. Em suma, para saber representar algo é preciso conhecê-lo.

Para dirimir essas dificuldades, Rêgo (2008) aponta a Educação Gráfica (EG) como fundamental na formação do arquiteto. A Educação Gráfica (EG) é definida por Rêgo (2008) como sendo o conjunto das "disciplinas de desenho" que compõem o currículo escolar dos cursos (básico, médio e superior) e que trabalham com conteúdos para o desenvolvimento da CV3D, da linguagem e da representação gráfica. A autora defende que se faz necessário buscar e desenvolver métodos e abordagens didáticas novas que trabalhem com as diversas mídias e tipos de representação, as quais possam dar suporte ao desenvolvimento do projeto de arquitetura.

A partir do exposto, o presente trabalho toma como problema de pesquisa a questão da dificuldade de visualização espacial dos estudantes no processo de representação gráfica do projeto arquitetônico. Para isso o trabalho estruturou-se a partir das teses de Tamashiro (2010) e de Rêgo (2008), relacionando a utilização do CTC em processos didáticos para potencializar o desenvolvimento da CV3D. Desse modo, este trabalho toma como hipótese que o uso do CTC como uma abordagem didática pode auxiliar a reduzir as dificuldades apresentadas pelos estudantes em representar graficamente os projetos de arquitetura.

Nesse sentido, foi realizado um experimento didático utilizando o CTC com os discentes do curso de Arquitetura e Urbanismo da Universidade Federal de Pernambuco, na disciplina eletiva de Tópicos Especiais em Geometria Gráfica 2DI (TE-GEO 2DI), ofertada a partir do terceiro período, integrando os conhecimentos relativos à computação gráfica aplicada ao projeto arquitetônico. $\mathrm{O}$ experimento exigiu que os estudantes realizassem o levantamento de escadas do ambiente real e representassem graficamente suas projeções mongeanas em meio digital, utilizando-se das regras da linguagem do desenho técnico arquitetônico. As vistas mongeanas se constituem como sendo o produto gráfico do chamado Sistema Mongeano, que foi elaborado por Gaspar Monge (1746-1818) com o intuito de resolver problemas relacionados ao projeto e à representação de objetos. O sistema inventado por Monge consiste numa dupla projeção cilíndrica ortogonal e é utilizado até os dias de hoje por profissionais que trabalham com a representação da Forma. Ele emprega planos perpendiculares entre si, sobre os quais são projetados ortogonalmente todos os pontos do objeto, resultando, assim, nas vistas mongeanas (Machado, 1976). 
Assim, o trabalho tem como objetivo verificar se o CTC contribui para a melhoria do desempenho dos estudantes na representação gráfica os projetos de arquitetura, potencializando o desenvolvimento da CV3D dos mesmos.

\section{Metodologia: o experimento didático}

O curso de Arquitetura e Urbanismo da UFPE teve sua estrutura curricular baseada em práticas de pedagogia ativa, cuja abordagem possibilita ações interdisciplinares e protagonismo discente. A Pedagogia Ativa possui um conjunto de métodos que engajam os alunos em suas atividades para além de seu papel como expectadores. Nesse tipo de abordagem o professor transcende o seu papel de emissor e passa a colaborar, em conjunto com os alunos, para o desenvolvimento das atividades (Santos, 2016). Dentre os métodos de Pedagogia Ativa, um dos mais utilizados é o Problem Based Learn (PBL). Esse método caracteriza-se por promover o processo de ensino- aprendizagem a partir de situações-problema vinculados à realidade profissional (Cararo; Sade Junior; Chagas, 2017).

Todas as disciplinas do curso estão divididas entre conceituais, instrumentais e integradoras, sendo as disciplinas de Representação Gráfica consideradas instrumentais e as de Projeto consideradas como núcleo integrador de cada semestre. Durante o curso as disciplinas conceituais e instrumentais são divididas em módulos ao longo dos semestres letivos e estão organizadas de modo a prover os conhecimentos necessários às disciplinas de projeto (Rêgo; Porto Carreiro, 2014).

A disciplina TE-GEO 2DI trabalha os conteúdos teóricos e práticos acerca da representação gráfica de projetos de arquitetura, urbanismo e paisagismo, utilizando os recursos dos softwares gráficos CAD (Computer Aided Design/Drawing). Ainda que seja de caráter eletivo, a disciplina tem como pré-requisitos outras que contemplam os conteúdos de geometria gráfica bidimensional e tridimensional, bem como de representação gráfica arquitetônica. Assim, ao ingressar na disciplina de TE-GEO 2DI considera-se que o discente já possui conhecimentos referentes à visualização espacial e às normas de representação gráfica de projetos de arquitetura.

O método utilizado para o desenvolvimento da disciplina foi o PBL, o qual é resolvido pelos estudantes a partir de conhecimentos prévios e também dos conhecimentos adquiridos com a orientação do professor ao longo da disciplina.

É importante ressaltar que ao longo do processo o professor deve atuar como um tutor, supervisionando e provocando os discentes a buscarem soluções diferentes para a mesma questão. Embora o método tenha como cerne a autonomia do estudante na construção do conhecimento, o professor deve participar dos debates, orientando e fornecendo materiais para que o problema seja resolvido (Ribeiro, 2005; Ribeiro, 2008). Desse modo, o exercício exigiu que os discentes trabalhassem de forma ativa e, em certa medida, aprendessem os conteúdos a partir das necessidades requeridas pelas soluções do problema.

O experimento proposto foi composto de três etapas, traduzidas em três exercícios:

(1) Exercício 1 (E1): consistiu no desenvolvimento da representação gráfica, em software CAD 2D, de uma residência unifamiliar. $O$ objeto a ser representado foi desenvolvido em disciplina do semestre anterior em nível de esboço, ou seja, não atendia a NBR 6492 (Associação Brasileira de Normas Técnicas, 1994). Portanto o produto desse exercício foi o desenvolvimento do conjunto de representações gráficas (planta baixa, cortes, fachadas, plantas de locação e coberta) a partir desse esboço. $\mathrm{O}$ exercício estimulou o estudante a representar 
graficamente, e de acordo com a NBR 6492, algo proveniente da sua própria concepção, distanciando-se das abordagens mais tradicionais nas quais o estudante reproduz representações de um projeto dado;

Exercício 2 (E2): consistiu na representação gráfica mongeana, em software CAD 2D, de uma das escadas existentes no edifício do Centro de Artes e Comunicação da UFPE. Para isso os estudantes realizaram um levantamento da escada por meio de esboços à mão livre e, por fim, a representaram graficamente em meio digital utilizando as normas técnicas, em especial a NBR 6492 (Associação Brasileira de Normas Técnicas, 1994). O objetivo do exercício foi utilizar a abordagem didática de Tamashiro (2010) baseada no CTC para o desenvolvimento da CV3D (Rêgo, 2008). Essa abordagem possibilita que o discente trabalhe com os conteúdos de representação gráfica digital a partir de uma experiência com o objeto concreto, nesse caso a circulação vertical do tipo escada. Esse exercício buscou distanciar o estudante das abordagens didáticas mais tradicionais nas quais é feita a reprodução de um objeto a partir de dados gráficos fornecidos em mídia analógica.

(3) Exercício 3 (E3): se constituiu na representação gráfica em planta baixa de reforma e de ambientação. Foi fornecido aos estudantes a planta baixa de um bangalô, que não atendia às especificações técnicas e foram solicitados três produtos: (a) planta legal: planta baixa em conformidade com a NBR 6492 (Associação Brasileira de Normas Técnicas, 1994); (b) planta de reforma: representação gráfica de uma reforma que considerasse o acréscimo de um dormitório ao edifício e a acessibilidade de todo o bangalô segundo a NBR 9050 (Associação Brasileira de Normas Técnicas, 2015) e; (c) planta humanizada: planta baixa da pós-reforma com elementos de cores, texturas e mobiliários visando a compreensão pelo público leigo. A finalidade desse exercício era fazer o estudante compreender que para cada tipo de representação há objetivos de comunicação distintos.

\subsection{Métodos e procedimentos}

Em termos gerais podemos classificar essa pesquisa como experimental que, segundo Gil (2008), caracteriza-se pela realização de um experimento considerando formas de controle e variáveis, bem como os efeitos dessas condicionantes na experimentação. As pesquisas que se utilizam desse tipo de abordagem têm como cerne limitar-se aos achados do experimento, diferenciando-se das abordagens de argumentação lógica, as quais se estruturam a partir de teorias consolidadas sem uma necessária comprovação prática (Marconi; Lakatos, 2011).

Tendo em vista o objetivo desse trabalho, que foi verificar se o CTC contribuiu para a melhoria do desempenho dos estudantes na representação gráfica em projetos de arquitetura, foi proposto um experimento realizado em três etapas. Sendo o CTC utilizado apenas na segunda etapa, permitindo a comparação entre desempenho e percepção, dos estudantes antes (E1) e depois (E3) da experiência com tal abordagem. A análise comparativa relacionada ao desempenho foi de caráter quantitativo, enquanto que a análise relativa à percepção foi qualitativa.

A análise de desempenho foi estruturada a partir da comparação entre os resultados do E2 com o E1; em seguida, do E2 com o E3, e, por último, do E3 com o E1. A comparação entre os exercícios teve como objetivo verificar se a aplicação do CTC se refletiu numa melhora direta nas notas. Sabe-se que o desempenho não está 
restrito apenas às notas, no entanto, essa é uma maneira objetiva de verificar a contribuição da aplicação de uma abordagem didática em um processo de ensinoaprendizagem. Para a realização dessas análises foram elaboradas tabelas com as notas individuais dos estudantes e a média da turma em cada exercício.

Quanto à análise relativa à percepção foi realizado um questionário, utilizando a plataforma do Google Forms, respondido em sala de aula pelos discentes após a finalização do E3. O questionário possui um total de 15 perguntas divididas em duas partes: 1) perfil do estudante e 2) avaliação do experimento. É importante salientar que os respondentes tiveram suas identidades resguardadas com o intuito de obter respostas espontâneas. Com o questionário foi possível verificar como os estudantes perceberam essa nova abordagem para assim poder identificar seus limites, dificuldades $\mathrm{e}$ potencialidades.

\section{Resultados e discussões}

A amostra dessa pesquisa é composta por 30 estudantes. Em relação ao perfil dos mesmos, $87,5 \%$ pertence ao $3^{\circ}$ período, enquanto que os $12,3 \%$ restante estão divididos igualmente entre $2^{\circ}, 4^{\circ}$ e $9^{\circ}$ períodos. Isso nos mostra que o grupo amostral é majoritariamente conformado por estudantes iniciantes do curso de graduação em Arquitetura e Urbanismo, indicando que a maior parte dos estudantes possui pouca experiência em representação arquitetônica. Desse grupo, 66,7\% teve aula de geometria no ensino básico e $25 \%$ realizou algum curso técnico, sendo $50 \%$ edificações, $16,7 \%$ eletrotécnica, $16,7 \%$ design de interiores e 16,7\% rede de computadores. Em todos os cursos técnicos foram ofertadas disciplinas de geometria gráfica, sendo a de Desenho Assistido por Computador (CAD) a que apresentou a maior porcentagem (100\%), seguido por Desenho Técnico (83,3\%) e Desenho Arquitetônico (66,7\%). Os 100\% dos estudantes que realizaram algum curso técnico declararam que essas disciplinas de geometria gráfica contribuíram para um melhor desempenho nas disciplinas de geometria gráfica do curso de graduação. Em suma, os dados dividem os estudantes em dois grupos: (1) composto por $75 \%$ da turma que possui pouca experiência em representação gráfica arquitetônica; (2) composto por $25 \%$ da turma que possui alguma experiência em representação gráfica devido às disciplinas do curso técnico. Demonstrando, conforme trata Rêgo (2008), a importância da EG na formação do arquiteto e urbanista.

A hipótese central da pesquisa é de que o uso do CTC como uma abordagem didática pode auxiliar a dirimir as dificuldades apresentadas pelos estudantes em representar graficamente os projetos de arquitetura. Para tanto, estruturou-se as seguintes sub-hipóteses:

1. O desempenho dos estudantes no E2 seria maior do que no E1, uma vez que se entende que o uso do CTC contribuiria para o processo de aprendizagem;

2. O desempenho dos estudantes no E3 seria maior do que no E1, porque espera-se que a experiência com o CTC no E2 se reflita, mesmo que indiretamente, nos processos subsequentes de aprendizagem, no caso o E3;

3. O desempenho dos estudantes no E2 seria maior do que no E3, pois entende-se que como o E2 conta com a aplicação direta do CTC o desempenho do estudante nessa atividade seria mais potencializado do que no E3.

É necessário esclarecer que para efeito de análise, quando o desempenho do estudante se mantém constante não é possível aferir mudança no desempenho dos mesmos, portanto foram retirados da amostra total o desempenho de dois estudantes. 
Os resultados mostraram que a primeira sub-hipótese foi confirmada, pois 92,86\% dos estudantes apresentaram desempenho mais elevado no E2 quando comparado com o os resultados do E1. Já a segunda sub-hipótese foi refutada, uma vez que apenas 32,14\% dos estudantes tiverem um desempenho maior no E3 em relação ao E1. Por fim, a terceira e última sub-hipótese foi confirmada, já que $89,28 \%$ dos estudantes obtiveram desempenho menor no E3 se comparado ao E2. Uma explicação possível para o resultado da análise da segunda sub-hipótese (refutada) é que há uma tendência que o desempenho sofra um declínio devido a motivos variados, em especial a sobrecarga de atividades no final do semestre. O principal aspecto a ser destacado nesse experimento é de que o uso do CTC, como uma abordagem didática, trouxe benefícios para o desenvolvimento da CV3D.

\subsection{Análise do Experimento: abordagem didática e mídia empregadas}

Ao serem questionados sobre qual exercício preferiram realizar, $75 \%$ declarou preferir representar uma escada existente, ou seja, um exercício utilizando a abordagem didática do CTC. Desse grupo, 62,5\% afirmou que a razão dessa escolha é a facilidade em realizar a tarefa. A partir desse dado, é possível perceber que a experiência com elementos concretos facilita as atividades que envolvem a representação gráfica arquitetônica, reforçando o próprio conceito do CTC como abordagem didática.

Quanto à mídia empregada (analógica ou digital) foi questionado quais dificuldades eles perceberam no seu uso. Sendo essa disciplina totalmente digital, foi utilizada a experiência dos estudantes em disciplinas anteriores no próprio curso, as quais eram analógicas, para fazer a comparação com a mídia digital. A interpretação dos dados sugere que os discentes não fazem distinção entre dificuldades de conteúdo e operacionais. Assim, para uma análise mais refinada, coube uma reflexão sobre o teor das dificuldades encontradas pelos estudantes, que foram classificadas em dois grupos:

(1) Dificuldades operacionais: se referem ao uso de procedimentos e ferramentas subjacentes ao processo de representação gráfica;

(2) Dificuldades de conteúdo: são relativas à construção do conhecimento necessário para realizar as representações arquitetônicas.

$\mathrm{Na}$ mídia digital as dificuldades foram: (1) operacionais: acionamento de comandos, plotagem e retrabalho, que somam 26,09\%; (2) de conteúdo: representação arquitetônica, detalhes construtivos, curvas, corrimão, tipos de linhas, vistas ortográficas e inclinação da escada, totalizando 56,52\%. Os demais estudantes $(17,39 \%)$ declarou não ter experenciado dificuldades. Já em relação à mídia analógica as dificuldades foram: (1) operacionais: cumprimento do prazo e precisão na representação das linhas, as quais somam 65\%; (2) de conteúdo: representação de degraus, tipos de linha, cortes e representação de ideias, totalizando 35\%. Conclui-se, a partir dos dados, que quando utilizam as mídias analógicas eles apresentam mais dificuldades operacionais do que contéudo. Já na utilização da mídia digital os discentes apresentam menos dificuldades operacionais e mais dificuldades de contéudos. Partindo do pressuposto de que quando há dificuldades de caráter operacional estas se sobrepõem às dificuldades de conteúdo, é possível tecer a seguinte interpretação: quando o estudante não possui problemas operacionais, independentemente da mídia, há uma tendência de que ele se dedique ao conteúdo.

Vale salientar que a aprendizagem do operacional, seja na mídia analógica ou digital, não pode se sobrepor à aprendizagem dos conteúdos de geometria gráfica no processo de EG do indivíduo. Entende-se aqui que o operacional se constitui apenas em uma ferramenta, ou seja, um meio para se chegar aos conteúdos. Por exemplo, quando 
o docente optar pela mídia digital é preciso deixar claro para os estudantes que os conhecimentos dessas disciplinas vão além da manipulação de softwares gráficos.

\subsection{Análise do Experimento: levantamento}

Ao serem questionados se já haviam realizado algum levantamento, 54,2\% declarou que sim, sendo que, desse grupo, 53,8\% o fez em disciplina anterior, 30,8\% em estágio e $15,4 \%$ em curso técnico. Quanto às dificuldades encontradas para a realização do levantamento foram encontrados os seguintes resultados: (1) dificuldades de conteúdo: saber o que medir (25\%) e construção de curvas e ângulos $(5 \%)$, somando 30\%; (2) dificuldades operacionais: acesso para medição (15\%), medição de elementos irregulares da escada (20\%) e ferramentas para medição (5\%), somando 40\%; (3) $30 \%$ não apresentou dificuldades. Dessa forma, percebe-se que para se trabalhar com o CTC é importante ter o cuidado prévio com questões operacionais, tais como: escolha de objeto com fácil acesso e esclarecimento sobre o uso das ferramentas para a realização do levantamento.

Os dados a seguir mostram que experiência prévia com levantamento arquitetônico pode ser comparado com a vivência que alguns estudantes têm com o CTC, já que essa abordagem tem como característica fundamental a experiência com objetos concretos. Nesse sentido, buscou-se compreender a relação que existe entre experiência prévia e dificuldades. De acordo com o experimento, verificou-se que $30 \%$ dos estudantes não apresentou dificuldades em realizar levantamentos arquitetônicos, sendo que desse grupo 62,5\% declarou possuir experiência prévia com tal atividade. Já em relação àqueles que declararam não saber o que medir (25\%), 60\% não teve experiência prévia com levantamento. Portanto, tais dados reforçam que a abordagem pelo CTC contribui para dirimir as dificuldades com as representações gráficas arquitetônicas. Ao mesmo tempo, verificou-se que $40 \%$ dos estudantes que possuiam experiência prévia com o CTC teve dificuldades em saber quais elementos construtivos medir. É possivel suscitar que a experiência prévia desses sujeitos não contemplou o conteúdo específico de escadas. Tal fato pode indicar uma possível limitação do CTC no tocante à especificidade do conteúdo tratado, ou seja, talvez o CTC não seja capaz de gerar contribuições genéricas à CV3D.

\subsection{Análise do Experimento: escadas}

Cerca de $90 \%$ dos discentes já havia realizado uma representação gráfica de escada sendo que, desse grupo, 95,4\% o fez em disciplina de representação gráfica anterior. Considerando a totalidade dos estudantes, 81,8\% declarou ter aprendido algo novo com o exercício proposto, enquanto $18,2 \%$ não obteve nenhum conhecimento novo. Quanto aos novos conhecimentos adquiridos, realizou-se uma pergunta aberta para identificar quais os novos conteúdos aprendidos no exercício proposto e obteve-se os seguintes resultados: como projetar escadas (20\%), desenvolver corte (15\%), detalhamento (10\%), aperfeiçoar representação de escada (10\%), elementos construtivos da escada (5\%), melhorar visão espacial (5\%), medidas principais da escada (5\%), escala (5\%), tipo de linha (5\%) e novos comandos do software CAD (20\%), salientando que este último trata-se de uma questão operacional. O E2 demostrou que os estudantes aprenderam melhor o conteúdo de representação a partir do CTC, visto que as questões relativas ao conteúdo somam $80 \%$. 


\section{Considerações Finais}

Este trabalho teve como objetivo principal investigar o uso do CTC como estratégia para dirimir as dificuldades de visualização espacial para estudantes de Arquitetura e Urbanismo no processo de representação gráfica do projeto arquitetônico. Para isso foi realizado um experimento com estudantes da UFPE, os quais realizaram levantamentos de escadas e representaram graficamente suas projeções mongeanas em meio digital, utilizando-se das regras do desenho técnico.

Os resultados mostram que, de maneira geral, o CTC contribuiu para o desenvolvimento da cacidade de visualização espacial e para o processo de projeto e suas representações gráficas. Isso pôde ser verificado analisando o rendimento dos estudantes nos três exercícios. O rendimento no exercício E2, o qual contou com o CTC, foi maior do que nos exercícios E1 e E3. No entanto, verificou-se que o CTC apresenta limitações uma vez que ele não garante o desenvolvimento de uma capacitação mais genérica no tocante à CV3D.

Com relação ao uso das mídias (digitais e analógicas) para as práticas de ensino de representação gráfica arquitetônica, verificou-se que quando o estudante não possui dificuldades operacionais, há uma tendência de que ele se dedique ao conteúdo. No mesmo sentido, quando os estudantes se deparam com uma dificuldade operacional isso representa um entrave no processo de ensino/aprendizagem. Em suma, para os estudantes, as questões operacionais são mais problemáticas do que as questões de conteúdo, quando essas últimas deveriam ser o foco.

$\mathrm{Na}$ abordagem digital os estudantes apresentaram mais dificuldades de conteúdo do que operacionais, enquanto que na analógica foi o inverso. Diante desse resultado esperava-se que a preferência dos discentes fosse pela mídia analógica. No entanto, todos declararam preferência pelo uso da mídia digital. Observou-se que a utilização da mídia digital propicia menor tempo de execução e maior controle da precisão, enquanto que na mídia analógica ocorre o oposto, maior tempo para execução das tarefas e menor controle da precisão. Nesse sentido, percebe-se que a aprendizagem dos conteúdos não foi a referência para a escolha da mídia. De acordo com os dados obtidos, o que balizou a escolha foram questões operacionais. Indicando que na percepção desse grupo de estudantes a prioridade está no cumprimento das atividades exigidas pela disciplina e não no conteúdo propriamente dito, ou seja, o conteúdo é sobrepujado às questões operacionais utilizadas para estudá-lo.

Os dados corroboram com o argumento de Lopes, Carneiro-da-Cunha, Gusmão, (2018), de que esse dissenso ocorre porque a Geometria Gráfica não é percebida como Ciência e nesse processo é confundida com o meio (operacional) que ela utiliza para estudar seu campo de conhecimento (conteúdo). A Geometria Gráfica tem como cerne estudar a forma e para isso utiliza-se de uma linguagem cuja ferramenta de expressão são as representações gráficas geométricas. Portanto, é possível afirmar a partir desse trabalho que a preferência dos estudantes incide sobre as mídias digitais, apesar de apresentarem mais dificuldade de conteúdo. Nesse sentido, é importante que as pesquisas se dediquem à investigação de soluções didático-metodológicas para o desenvolvimento das atividades em mídia digital.

\section{Referências Bibliográficas}

ASSOCIAÇÃO BRASILEIRA DE NORMAS TÉCNICAS. NBR 6492: representação de projetos de arquitetura. Rio de Janeiro, 1994. 
ASSOCIAÇÃO BRASILEIRA DE NORMAS TÉCNICAS. NBR 9050: acessibilidade a edificações, mobiliário, espaços e equipamentos urbanos. Rio de Janeiro, 2015.

CARARO, J.; SADE JUNIOR, W. ; CHAGAS, A. Prática de ensino em uma disciplina do curso de arquitetura e urbanismo. In: XIII Congresso Nacional de Educação, IV Seminário Internacional de Representações Sociais, Subjetividade e Educação e VI Seminário Internacional sobre Profissionalização Docente. Curitiba, 2017.

CHING, Francis D. K. Desenho para arquitetos. Francis D. K. Ching, Steven P. Juroszek; tradução técnica: Alexandre Salvaterra. 2. ed. Porto Alegre: Bookman, 2012.

FARELLY, L. Fundamentos da Arquitetura. Porto Alegre: Bookman, 2014. 200p.

LOPES, A. V. de F.; GUSMÃO, M. B. R. de; CARNEIRO-DA-CUNHA, M. Quem somos? uma abordagem epistemológica sobre a geometria gráfica e suas práticas. Revista Geometria Gráfica. Recife. Ano 2018, Vol. 2, n.1.

MACHADO, Ardevan. Geometria Descritiva: teoria e exercícios. São Paulo: McGraw-Hill do Brasil Ltda, 1976.

MARCONI, M. A.; LAKATOS, E. M. Fundamentos de metodologia científica. São Paulo: Atlas, 2011.

MONTENEGRO, G. Desenho Arquitetônico. 4a ed. São Paulo:Edgar Blucher, 2001. MONTENEGRO, G. Desenho de projetos. São Paulo: Edgar Blucher, 2007.

RÊGO, R. Educação gráfica para o processo criativo projetual arquitetônico: as relações entre a capacidade visiográfica tridimensional e a utilização de instrumentos gráficos digitais para a modelagem geométrica. 320p. Tese (Doutorado) - Universidade Federal da Bahia, Programa de Pós-Graduação em Arquitetura e Urbanismo, 2008.

RÊGO, R; PORTO CARREIRO, P. Fundamentos da gestão de informação projetual em curso de arquitetura e urbanismo. In: Encontro brasileiro de tecnologia de informação e comunicação na construção, 7, 2015, Recife. Anais... Porto Alegre: ANTAC, 2014.

RIBEIRO, L. A aprendizagem baseada em problemas (PBL) na educação em engenharia. Revista de Ensino de Engenharia, v. 27, n. 2, p. 23-32, 2008.

. A aprendizagem baseada em problemas (PBL): uma implementação na Educação em Engenharia da voz dos atores. 209 p. Tese (Doutorado)-Universidade Federal de São Carlos, Programa de Pós-Graduação em Educação, 2005.

SANTOS, A. Um Método de Aprendizagem Baseada em Desafios: um estudo de caso em ambientes de desenvolvimento de aplicativos. 179p. Tese (Doutorado) Pontifícia Universidade Católica do Rio Grande do Sul, Programa de Pós- Graduação em Ciência da Computação, 2016.

TAMASHIRO, H. A. Entendimento técnico-construtivo e desenho arquitetônico: uma possibilidade de inovação didática. 210 p. Tese (Doutorado) - Escola de Engenharia de São Carlos da Universidade de São Paulo. Programa de Pós-Graduação em Arquitetura e Urbanismo, 2010.

ZEVI, Bruno. Saber ver a arquitetura. São Paulo, Ed. Martins Fontes, 1996. 